\title{
Role of Biomarkers in Sports: Review
}

\author{
Monika
}

\begin{abstract}
Aim: The aim of this review was to discuss the role of biomarkers in sports. Results: The sophisticated technologies can now be used to study subtle changes in biochemical modifications following physical exercise. Conclusion: Rapid advancements in molecular techniques have enabled the establishment of alterations in the concentration bio molecules following physical exercise.
\end{abstract}

\author{
Monika \\ Assistant Professor \\ Department of Biotechnology \\ Mata Gujri College, Fatehgarh Sahib, Punjab, India \\ E-mail: monika187@ rediffmail.com
}

\author{
Key Words: Athlete , Biomarkers, \\ Inflammation, Performance
}

DOI: $10.18376 / j e s p / 2017 / v 13 / \mathbf{i} 2 / 111287$

\begin{abstract}
A biomarker, or biological marker, is in general a substance used as an indicator of a biological state. In medicine, a biomarker is a measurable characteristic that reflects the severity or presence of some disease state. More generally a biomarker is anything that can be used as an indicator of a particular disease state or some other physiological state of an organism. Biomarker levels represent a summation of the influence of acute and chronic comorbidities

Proteins, metabolites, electrolytes, and other small molecules may serve as biomarkers for athletes and recreationally active individuals. Advances in big data approaches to assessing health and performance of athletes suggest that using the newest technology with intrinsic data such as biochemical, hematological data can be powerful in identifying the balance between training and recovery in each unique individual. Indeed, many commercially available services are offering biochemical and genetic testing for athletes, and professional athletes are reportedly exploiting technology and biomarker testing to track performance and recovery during training. Numerous biomarkers may assess different aspects of health, sport performance, and recovery. Biomarker testing/ analysis poses many challenges: (a) single biomarkers are not definitive for diagnosing broad physiological function such as "recovery" in sport, (b) sensitivity of single biomarkers to detect overtraining or injury risk is limited, (c) reference ranges for athletes and specific subgroups of athletes are not well defined, (d) inter-individual variance in absolute values and relative changes in biomarkers, and (e) highly contextualized nature of biomarker testing and analysis. A single measurement of a biomarker does not allow for precise determination of an individual's health status. For example, although the immune signaling molecule interleukin-6 (IL-6) is a cytokine that can indicate inflammation alone, it provides little diagnostic information about chronic inflammation during overtraining in an athlete; it has both pro inflammatory and anti-inflammatory roles and responds to many stimuli acutely and chronically. There seems to be evidence for the usefulness of IL-6 as a potential biomarker of overtraining. However, researchers agree that multiple cytokines should be measured together when attempting to detect chronic inflammation in athletes, and that other variables related to physiological/physical function and upstream stimuli for
\end{abstract}


chronic inflammation should be measured simultaneously (MacKinnon 2000; Smith 2000). Data on multiple inflammatory cytokines, endocrine markers of long-term dysregulation and overtraining like testosterone and cortisol, and muscle damage markers like creatine kinase (CK) can be integrated to provide precise and accurate information about an athlete's health and overtraining status. Relying on a single marker to sensitively and precisely detect overtraining is overly simplistic given the pleiotropic nature of most biological markers. The markers of hydration state, nutrition/metabolic health, oxygen transport, muscle status, inflammation, injury risk, and food allergies that can be integrated to help athletes interpret their blood biomarker data to meaningful practical application. Further complicating biomarker analysis is the fact that isolated or infrequent testing of biomarkers provides limited information. There are few athlete-specific reference ranges for most biomarkers, and this is in part because there is large inter-individual variance in biomarker values, and that measurement of biomarkers can vary by context. Again considering the example of overtraining biomarkers, people generally exhibit high variability in serum/plasma cytokine levels and responsiveness ( $\mathrm{Li}$ et al. 2016; Kleiner et al. 2013; Todd et al. 2013) and athletes could exhibit greater rates of variability or different ranges of values (compared with average/sedentary individuals) entirely as they do for other markers such as muscle damage marker CK (Mougios 2007). Markers such as the many inflammatory cytokines are elevated after exercise in healthy individuals and return to baseline values within minutes to hours after exercise (Suzuki et al.2002). Absolute values of cytokines in a one-time blood sample might be meaningful if values are elevated or decreased outside of the large range of inter-individual variability observed, but perhaps more meaningful might be the responsiveness of circulating cytokine levels to a challenge such as an acute training bout or weeks of training. The absolute resting levels of biomarkers may not change while the response to stress could be abnormal. Thus, timing of the measurement and an individual's average resting levels over multiple days is relevant to interpretation and important to understanding the normal fluctuation in biomarkers for a given individual over a short period of time and in response to exercise and recovery over the course of hours, days, or weeks. Time course for when to take measurements and how frequently are included in the discussion of specific biomarkers. Although literature do not recommend a precise testing schedule that is suitable for all athletes under any training conditions, but it can recommend four main considerations for determining frequency/timing of biomarker testing. The first recommendation is to test at the beginning and end the key points of training transitions. For example, testing before and at the end of preseason training will provide important information about the athlete coming out of off-season or rest periods and how preseason training has prepared the athlete for the competitive season without ideally, inducing any overtraining or injury. Second, it is recommended that during the competitive season, which may have training sub-cycles, that biomarker testing be completed around a single bout of exercise. Testing can be administered before and after (a) a bout of exercise during a particularly challenging training week, (b) a performance test, or (c) a bout of exercise after recovery from an injury or after some shift in training. This type of testing will elucidate any deficiencies or defects in biomarker responses to an acute stress. This would be valuable when resting values of biomarkers might not reveal any concerns, but the response and recovery from a bout of exercise would more sensitively detect concerns. A third recommendation is to test before and multiple times after a major competition event or injury. In this case, there is a severe stress imposed by either the competitive event or an injury and biomarker testing multiple times after the event will allow an athlete to determine whether recovery has occurred on a biochemically measurable level. This case highlights the potential of biomarker testing to precisely detect potential health/recovery concerns when an athlete might feel ready, but may not actually be ready at the tissue/cellular level. Finally, a recommendation to establish standards for each individual and address the variability in most biomarkers, is that biomarker testing be done on multiple days 
during off-season when an athlete is fit, healthy, and rested to determine the athlete's average resting values for all biomarkers to be tested under training conditions. Flexibility should be built into biomarker testing schedules to account for testing that can be associated with an athlete's subjective feelings of fatigue, measurable decreases in performance, and injury incidents. Accurately and precisely assessing health and performance of athletes requires a more comprehensive, integrative, and dynamic approach to biomarker analysis. A simplistic approach to using molecular biology/biochemistry in applied/practical sport science will not be appropriate in maximizing the benefit of biomarker testing to diagnose and make training decisions. The application of biomarker testing to traditional sport assessment/coaching requires thoughtful selection of multiple biomarkers and schedule of biomarker testing, and informed interpretation of both biochemical results and physiological/physical data about athletes. The present review present an example of holistic approach to tracking athletes using biomarkers that assess nutritional health, metabolic health, hydration status, muscle status, endurance performance, injury status and risk, and inflammation. Diet and training affect these key aspects of health and performance that can be assessed with biomarkers that have been relatively well studied; examples of evidence based biomarkers for each specific aspect of health/performance are suggested based on review of the literature. The literature suggests ideally, a comprehensive approach to biomarker analysis, but markers are presented based on their respective physiological relevance for individuals seeking a more focused approach to hematological assessment.

\section{References}

MacKinnon, LT. Special feature for the Olympics: Effects of exercise on the immune system: Overtraining effects on immunity and performance in athletes. Immunol Cell Biol 78: 502-509, 2000.

Smith, LL. Cytokine hypothesis of overtraining: A physiological adaptation to excessive stress? Med Sci Sports Exerc 32: 317-331, 2000.

Kleiner, G, Marcuzzi, A, Zanin, V, Monasta, L, and Zauli, G. Cytokine levels in the serum of healthy subjects. Mediators Inflamm 2013: 434010, 2013.

Li, Y, Oosting, M, Deelen, P, Rican o-Ponce, I, Smeekens, S, Jaeger, M, Matzaraki, V, Swertz, MA, Xavier, RJ, Franke, L, Wijmenga, C, Joosten, LA, Kumar, V, and Netea, MG. Interindividual variability and genetic influences on cytokine responses to bacteria and fungi. Nat Med 22: 952-960, 2016.

Todd, J, Simpson, P, Estis, J, Torres, V, and Wub, AH. Reference range and short- and long-term biological variation of interleukin (IL)-6, IL-17A and tissue necrosis factor-alpha using high sensitivity assays. Cytokine 64: 660-665, 2013.

Mougios, V. Reference intervals for serum creatine kinase in athletes. Br J Sports Med 41: 674-678, 2007.

Suzuki, K, Nakaji, S, Yamada, M, Totsuka, M, Sato, K, and Sugawara, K. Systemic inflammatory response to exhaustive exercise. Cytokine kinetics. Exerc Immunol Rev 8: 6-48, 2002. 